\title{
Selection criteria for targets of asteroseismic campaigns
}

\begin{abstract}
F. P. Pijpers ${ }^{\star}$
Theoretical Astrophysics Center (TAC), Department of Physics and Astronomy (IFA), Aarhus University, Ny Munkegade, 8000 Århus C, Denmark

Received 8 November 2002 / Accepted 10 December 2002

Abstract. Various dedicated satellite projects are underway or in advanced stages of planning to perform high-precision, long duration time series photometry of stars, with the purpose of using the frequencies of stellar oscillations to put new constraints on the internal structure of stars. It is known (cf. Brown et al. 1994) that the effectiveness of oscillation frequencies in constraining stellar model parameters is significantly higher if classical parameters such as effective temperature and luminosity are known with high precision. In order to optimize asteroseismic campaigns it is therefore useful to select targets from among candidates for which good spectroscopic and astrometric data already exists. This paper presents selection criteria, as well as redeterminations of stellar luminosity and reddening for stars satisfying these criteria.
\end{abstract}

Key words. stars: oscillations - stars: evolution - stars: fundamental parameters

\section{Introduction}

The aim of asteroseismology is to use the properties of the oscillations of stars to place constraints on their internal structure. The frequencies of stellar oscillations can be used to determine parameters of stars that are either inaccessible to classical observation or can not be determined with an accuracy that is sufficient for many purposes. The foremost examples of these parameters are the mass and angular momentum of stars. Currently, accurate masses of stars can only be determined if they are members of close binary systems. Even for the best spectroscopic data and stellar atmospheres modelling currently available, for single stars their mass is not known to better than $20 \%$. If one wishes to determine the mass function of stars and investigate its universality, for instance in order to investigate star formation processes, the current data are heavily biased to stars that have formed in binary systems, which may not reflect the variety of conditions under which stars can form and thus provide an incorrect mass function for the galaxy. As a consequence of the fact that forming stars contract from molecular clouds many orders of magnitude larger than their main sequence stellar radius, a substantial amount of angular momentum must be shed during the contraction. An important constraint for theories of mechanisms mediating this angular momentum loss would be the measurement of the angular momentum of stars for a range of masses. Also, for the post-main sequence evolution of in particular high-mass stars the loss of angular momentum during evolution is similarly important. Unfortunately spectroscopic observations have access to only the projected surface rotation velocity. The true angular momentum can only be obtained through asteroseismology (Pijpers 2003).

\footnotetext{
* e-mail: fpp@phys.au.dk
}

Oscillation frequencies are measures of sound travel time through the star, which is an average of the inverse of the sound speed. There is a relation between the sound speed and the mass because stars are in hydrostatic equilibrium. There is a secondary dependence on hydrogen abundance which arises through the change in the mean atomic mass on which the sound speed depends. Thus if a sufficient number of modes is present and identified in the time series of an oscillating star, it should in principle be possible to measure its mass, its core hydrogen abundance ("age"), and its rotation rate. Using more sophisticated inverse analyses other information, such as depths of convection zones, might be possible to extract as well. The identification of observed modes of oscillation and the subsequent analysis is considerably facilitated if 'external' constraints such as a stellar effective temperature or a stellar radius are available (cf. Brown et al. 1994).

Section 2 of this paper discusses the stars that have been selected as the most appropriate candidates for observation in the MONS experiment (Measuring Oscillations in Nearby Stars) on board the Rømer satellite which is an element in the Danish small satellite program (Christensen-Dalsgaard 2002). Relevant stellar parameters from the literature or derived in the paper are presented here. Section 3 presents a set of selection criteria which can easily be generalized for other missions with different limiting sensitivities. These selection criteria are intended to maximize the effectiveness of any oscillation frequencies obtained in constraining stellar structure. Section 4 presents some results and discussion.

\section{The short-list of stars for MONS}

For the MONS mission a short-list of stars has been decided upon from which a subset of around 20 stars is to be selected for observation by the main camera. In making this list, a 
variety of criteria were used in order to obtain a set of stars which are representative of several classes or groups corresponding to the interests of a large international community. All of these stars are solar-like in the sense that they are expected to exhibit oscillations driven stochastically by convection in a surface convection zone. It is desirable to have stars representative of several subgroups within this class: close solar analogues in mass as well as somewhat lower and higher mass stars (at various stages of evolution), some stars at lower and higher metallicity than the Sun, some stars that on the basis of the level of their magnetic activity are expected to rotate rather more rapidly than the Sun and finally a few stars at higher effective temperature.

However it is useful to consider whether it is possible to create a larger and possibly more uniform sample of stars using quantitative criteria. Such a list would include most of the stars listed above but could serve as resource for other asteroseismic missions and observing campaigns as well as for contingency planning for the MONS mission itself. In order to do this it is first necessary to obtain the relevant stellar parameters. The luminosity $L$ in units of the solar value $L_{\odot}$ can be obtained from measurable quantities using the following equation:

$$
\begin{aligned}
\log \frac{L}{L_{\odot}}= & 4.0+0.4 M_{\mathrm{bol}, \odot}-2.0 \log \pi[\mathrm{mas}] \\
& -0.4\left(V-A_{V}+B C(V)\right) .
\end{aligned}
$$

Here and throughout the paper logarithms are taken to base 10 . The parallax $\pi$ is obtained from the Hipparcos (ESA 1997) satellite in all cases except for a few binary systems. For these the value is used that is obtained by Söderhjelm (1999) which takes into account corrections due to the relative motion of the two components in their orbit. The Johnson $V$ magnitude is obtained from the Catalogue of Photometric Data (Mermilliod et al. 1997). For most stars in the MONS shortlist there are high precision determinations of the stellar parameters: effective temperature, surface gravity, and metallicity $\left(T_{\text {eff }}, \log g,[\mathrm{Fe} / \mathrm{H}]\right)$ directly from spectroscopy. For the remaining stars spectroscopy is being obtained and will be reported elsewhere (Bruntt 2003). With these values it is possible to obtain colours $(B-V)$, extinction $A_{V}$ and bolometric corrections $B C(V)$ on $V$ using the BaSeL library of model atmospheres (Lejeune et al. 1998). $M_{\text {bol, } \odot}$ is the solar bolometric absolute magnitude.

In order to be fully consistent, in principle it would be necessary to re-reduce the original spectra or obtain new ones and perform fitting for $\left(T_{\text {eff }}, \log g,[\mathrm{Fe} / \mathrm{H}]\right)$ using the same model atmosphere code that would also be used to calculate the colour and bolometric corrections. In practice this is not feasible for a large number of stars and therefore it is assumed that the uncertainties on the values for $\left(T_{\mathrm{eff}}, \log g,[\mathrm{Fe} / \mathrm{H}]\right)$ quoted by the various authors are sufficiently large to encompass any systematic effects between the various codes currently in use for spectral line fitting for these parameters. One possible systematic effect is changes in colours and bolometric corrections that can arise due to different assumptions concerning properties of convection. In order to asses the magnitude of such effects the bolometric corrections and colours were compared with those resulting from using model atmospheres of Bessel et al. (1998) with and without overshoot. For all stars in the MONS short-list the differences between the two treatments and between that grid and the BaSeL grid of model atmospheres were smaller than any differences introduced due to the uncertainty in the measurements of $\left(T_{\mathrm{eff}}, \log g,[\mathrm{Fe} / \mathrm{H}]\right)$. Of course very different treatments of convection such as proposed by Canuto \& Dubovikov (1998), or 3-D simulations (cf. Asplund et al. 1999) might well introduce substantial changes both in the estimation of $\left(T_{\text {eff }}, \log g,[\mathrm{Fe} / \mathrm{H}]\right)$ as well as in $B C(V)$ and $(B-V)_{0}$, but as yet such information is not available for large numbers of stars.

A summary of the stellar parameters is presented in Tables 1 and 2. In a number of cases more than one independent determination of $\left(T_{\text {eff }}, \log g,[\mathrm{Fe} / \mathrm{H}]\right)$ is available in the literature. However, in many references no error estimates are quoted, which makes it difficult to asses whether or not the various determinations are consistent. The values in Tables 1 and 2 are either from a reference which quotes an error estimate or the most recent determination available. The bolometric corrections on the $V$ magnitude $B C(V)$ are obtained by tri-linear interpolation in $T_{\text {eff }}, \log g$, and $[\mathrm{Fe} / \mathrm{H}]$ on the tables of Lejeune et al. (Lejeune et al. 1998) for the BaSeL library of model atmospheres. In order to be consistent with these tables, the bolometric magnitude of the Sun $M_{\mathrm{bol}, \odot}$ is taken to be 4.746 (Lejeune et al. 1998). An estimate of the uncertainty in the bolometric correction is obtained by treating the error estimates on $T_{\text {eff }}, \log g$, and $[\mathrm{Fe} / \mathrm{H}]$ as independent errors and combining them quadratically. The necessary partial derivatives of the bolometric correction with respect to each of these parameters are also obtained from the tables calculated with the BaSeL library of model atmospheres. For those stars for which no measurement error for $T_{\mathrm{eff}}, \log g$, and/or $[\mathrm{Fe} / \mathrm{H}]$ is available the error has been set to $100 \mathrm{~K}, 0.2$ and 0.1 respectively, which in some cases contributes substantially to the uncertainty in the bolometric correction and the intrinsic colour and therefore also to the error estimates for $L$ and $A_{V}$.

Since the stars MONS short-listed stars are all nearby, the visual extinction $A_{V}$ is expected to be very small. In fact in deriving relation (2) from observations it is often assumed that there is no extinction within $70 \mathrm{pc}$ of the Sun. Nevertheless in terms of the uncertainty estimate for the luminosity, in principle the term must be taken into account. To obtain an estimate of $A_{V}$ the standard relation between extinction and reddening is used (cf. Neckel et al. 1980):

$A_{V}=3.1\left[(B-V)-(B-V)_{0}\right]$,

where the intrinsic colour $(B-V)_{0}$ is obtained by tri-linear interpolation in $T_{\text {eff }}, \log g$, and $[\mathrm{Fe} / \mathrm{H}]$ on the tables of Lejeune et al. (Lejeune et al. 1998) for the BaSeL library of model atmospheres. The uncertainty in $(B-V)_{0}$ is obtained from the BaSeL library in the same way as it is for the bolometric correction. This error is then combined quadratically with the measurement error in $(B-V)$ to obtain an error estimate on the visual extinction.

The resulting values for the luminosity $L$ and visual extinction $A_{V}$ are listed in Table 2. In some cases negative values for the visual extinction are obtained, which can be due to measurement uncertainties for $(B-V)$ or the uncertainty in $(B-$ $V)_{0}$ propagated from the uncertainties in $\left(T_{\text {eff }}, \log g,[\mathrm{Fe} / \mathrm{H}]\right)$. 
Table 1. Properties of the short-listed stars for the MONS main camera ordered by Hipparcos number. Column 1: Hipparcos no.; 2: name; 3, 4: spectral type, source; 5, 6: known multiplicity, source; 7, 8, 9: parallax, $1 \sigma$, source; 10, 11, 12: effective temperature, $1 \sigma$, source; 13, 14: surface gravity, $1 \sigma$, source in Col. $12 ; 15,16:[\mathrm{Fe} / \mathrm{H}]-[\mathrm{Fe} / \mathrm{H}]_{\odot}, 1 \sigma$, source in Col. 12.

\begin{tabular}{|c|c|c|c|c|c|c|c|c|c|c|c|c|c|c|c|}
\hline \multirow{2}{*}{\begin{tabular}{|c|} 
HIP \\
2021
\end{tabular}} & \multirow{2}{*}{$\begin{array}{l}\text { name } \\
\beta \mathrm{Hyi}\end{array}$} & \multicolumn{2}{|l|}{ Spec.type $^{\mathrm{a}}$} & \multicolumn{2}{|c|}{ Mult. $^{a, b}$} & \multicolumn{2}{|c|}{$\begin{array}{c}\pi \\
{[\mathrm{mas}]}\end{array}$} & \multirow{2}{*}{$\begin{array}{r}\text { ref. }^{a} \\
2\end{array}$} & \multicolumn{2}{|c|}{$\begin{array}{c}T_{\text {eff }} \\
{[\mathrm{K}]}\end{array}$} & \multirow{2}{*}{$\begin{array}{r}\text { ref. }^{a} \\
9\end{array}$} & \multicolumn{2}{|c|}{$\begin{array}{c}\log g \sigma_{g} \\
{\left[\mathrm{~cm} / \mathrm{s}^{2}\right]}\end{array}$} & \multirow{2}{*}{$\begin{array}{c}\mathrm{Fe} / \mathrm{H}] \\
-0.11\end{array}$} & \multirow{2}{*}{$\begin{array}{r}\sigma_{[\mathrm{Fe} / \mathrm{H}]} \\
0.07\end{array}$} \\
\hline & & G2IV & 1 & & & 133.78 & 0.51 & & 5860 & 70 & & 4.05 & 0.30 & & \\
\hline 3821 & $\eta$ Cas & F9V & 1 & M & 4 & 167.99 & 0.62 & 2 & 5848 & 100 & 11 & 4.40 & 0.05 & -0.27 & 0.03 \\
\hline 7513 & $v$ And & F8V & 1 & M & 4 & 74.25 & 0.72 & 2 & 6135 & 61 & 8 & 4.08 & 0.18 & 0.11 & 0.09 \\
\hline 8102 & $\tau$ Cet & G8V & 1 & B & 4 & 274.17 & 0.80 & 2 & 5264 & 100 & 11 & 4.36 & 0.05 & -0.50 & 0.03 \\
\hline 8796 & $\alpha \operatorname{Tri}$ & F6IV & 1 & M & 4 & 50.87 & 0.82 & 2 & 6288 & & 16 & 3.91 & & 0.00 & \\
\hline 12777 & $\theta$ Per & F8V & 1 & M & 4 & 89.03 & 0.79 & 2 & 6248 & 80 & 20 & 4.20 & 0.10 & -0.01 & 0.07 \\
\hline 14632 & $\iota$ Per & G0V & 1 & B & 4 & 94.93 & 0.67 & 2 & 5989 & 61 & 8 & 4.19 & 0.21 & 0.16 & 0.09 \\
\hline 14879 & $\alpha$ For & F8V & 1 & B & 4 & 70.86 & 0.67 & 2 & 6000 & & 17 & & & -0.35 & 0.07 \\
\hline 15457 & $\kappa^{1} \mathrm{Cet}$ & $\mathrm{G} 5 \mathrm{Vv}$ & 1 & M & 4 & 109.18 & 0.78 & 2 & 5576 & 61 & 8 & 4.41 & 0.21 & 0.03 & 0.09 \\
\hline 16537 & $\epsilon$ Eri & $\mathrm{K} 2 \mathrm{~V}$ & 1 & $\mathrm{~B}$ & 4 & 310.75 & 0.85 & 2 & 5052 & 100 & 11 & 4.57 & 0.05 & -0.15 & 0.03 \\
\hline 17378 & $\delta$ Eri & K0IVe & 1 & & & 110.58 & 0.88 & 2 & 4884 & 61 & 8 & 3.40 & 0.18 & -0.11 & 0.09 \\
\hline 19893 & $\gamma$ Dor & F4III & 1 & & & 49.26 & 0.50 & 2 & 7300 & & 21 & 4.2 & & & \\
\hline 22449 & $\pi^{3}$ Ori & F6V & 1 & B & 4 & 124.60 & 0.95 & 2 & 6482 & 61 & 8 & 4.35 & 0.18 & 0.05 & 0.09 \\
\hline 27072 & $\gamma$ Lep A & F6V & 1 & M & 4 & 111.49 & 0.60 & 2 & 6302 & 61 & 8 & 4.26 & 0.18 & -0.05 & 0.09 \\
\hline 27913 & $\chi^{1}$ Ori & G0V & 1 & $\mathrm{~B}$ & 1 & 115.43 & 1.08 & 2 & 5869 & 61 & 8 & 4.45 & 0.18 & -0.01 & 0.09 \\
\hline 32362 & $\xi \mathrm{Gem}$ & F5III & 1 & & & 57.02 & 0.83 & 2 & 6464 & 100 & 23 & 3.81 & 0.02 & 0.00 & 0.01 \\
\hline 37279 & $\alpha$ CMi A & F5IV-V & 1 & M & 4 & 285.93 & 0.88 & 2 & 6500 & 80 & 10 & 4.04 & 0.10 & 0.00 & 0.05 \\
\hline 50954 & HR 4102 & F2IV & 1 & & & 61.67 & 0.49 & 2 & 7320 & 260 & 24 & & & & \\
\hline 55642 & $\iota$ Leo & F4IV & 1 & $\mathrm{~B}$ & 4 & 42.6 & 1.3 & 2 & 6739 & & 16 & 3.98 & & 0.06 & \\
\hline 57757 & $\beta$ Vir & F9V & 1 & B & 4 & 91.74 & 0.77 & 2 & 6109 & 100 & 11 & 4.20 & 0.05 & 0.17 & 0.03 \\
\hline 67927 & $\eta$ Boo & G0IV & 1 & B & 4 & 88.17 & 0.75 & 2 & 6003 & 61 & 8 & 3.62 & 0.18 & 0.25 & 0.09 \\
\hline 70497 & $\theta$ Boo & F7V & 1 & B & 4 & 68.63 & 0.56 & 2 & 6227 & 61 & 8 & 3.84 & 0.18 & -0.27 & 0.09 \\
\hline 71681 & $\alpha$ Cen B & K1V & 1 & B & 4 & 747.1 & 1.2 & 26 & 5255 & 50 & 7 & 4.51 & 0.08 & 0.24 & 0.03 \\
\hline 71683 & $\alpha$ Cen A & G2V & 1 & B & 4 & 747.1 & 1.2 & 26 & 5830 & 30 & 7 & 4.34 & 0.05 & 0.25 & 0.02 \\
\hline 71957 & $\mu \mathrm{Vir}$ & F2III & 1 & & & 53.54 & 0.95 & 2 & 7140 & 160 & 22 & & & & \\
\hline 76976 & HD 140283 & sdF3 & 3 & & & 17.44 & 0.97 & 2 & 5687 & 100 & 11 & 3.55 & 0.05 & -2.53 & 0.03 \\
\hline 77257 & $\lambda \mathrm{Ser}$ & G0V & 1 & B & 5 & 85.08 & 0.80 & 2 & 5915 & 100 & 11 & 4.10 & 0.05 & -0.01 & 0.03 \\
\hline 78072 & $\gamma \mathrm{Ser}$ & F6V & 1 & M & 4 & 89.92 & 0.72 & 2 & 6249 & 61 & 8 & 4.16 & 0.18 & -0.15 & 0.09 \\
\hline 81693 & $\zeta$ Her & G0IV & 1 & B & 4 & 93.7 & 0.60 & 26 & 5825 & & 14 & 3.80 & & 0.00 & \\
\hline 84405 & 36 Oph A & K0V & 1 & M & 4 & 167.08 & 1.07 & 2 & 5143 & & 19 & 4.60 & & -0.39 & \\
\hline 86974 & $\mu$ Her & G5IV & 1 & M & 4 & 119.05 & 0.62 & 2 & 5411 & 100 & 11 & 3.87 & 0.05 & 0.16 & 0.03 \\
\hline 88601 & 70 Oph A & KOV & 1 & B & 4 & 195.7 & 0.9 & 26 & 5260 & & 18 & 5.00 & & -0.25 & 0.15 \\
\hline 89937 & $\chi$ Dra & F7V & 1 & B & 4 & 124.37 & 0.52 & 26 & 6008 & 61 & 8 & 4.36 & 0.18 & -0.33 & 0.10 \\
\hline 99240 & $\delta \mathrm{Pav}$ & G6-8IV & 1 & & & 163.73 & 0.65 & 2 & 5538 & & 13 & 3.80 & & 0.28 & \\
\hline 102485 & $\psi$ Cap & F4V & 1 & & & 68.16 & 0.91 & 2 & 6632 & & 15 & 4.50 & & -0.11 & \\
\hline 104043 & $\alpha$ Oct & F4III & 25 & $\mathrm{~B}$ & 6 & 22.07 & 0.57 & 2 & & & & & & & \\
\hline 105858 & $\gamma \mathrm{Pav}$ & F6V & 1 & & & 108.50 & 0.59 & 2 & 6139 & 70 & 9 & 4.34 & 0.30 & -0.67 & 0.07 \\
\hline 109176 & $\iota$ Peg A & F5V & 1 & B & 4 & 85.06 & 0.71 & 2 & 6413 & 61 & 8 & 4.16 & 0.18 & 0.00 & 0.09 \\
\hline 110618 & $v$ Ind & A3V & 1 & B & 4 & 34.60 & 0.60 & 2 & 5381 & & 12 & 3.43 & & -1.34 & 0.08 \\
\hline 114996 & $\gamma$ Tuc & F1III & 1 & & & 45.40 & 0.61 & 2 & 6541 & & 16 & 3.88 & & -0.27 & \\
\hline
\end{tabular}

a Refs.: 1 = Hoffleit \& Warren Jr., Bright Star Cat. (1991), 2 = The Hipparcos and Tycho Cat. (1997), $3=$ Cayrel de Strobel et al., Cat. of [Fe/H] of F, G, K stars (2001), 4 = Worley et al., The Washington Visual Double Star Cat. (1996), $5=$ Abt \& Levy (1976), 6= Halbwachs (1981), $7=$ Neuforge et al. (1997), $8=$ Cenarro et al. (2001), $9=$ Castro et al. (1999), $10=$ Mashonkina $\&$ Gehren $(2000), 11=$ Soubiran et al. (1998), $12=$ Gratton et al. (2000), $13=$ Abia et al. (1988), $14=$ Cunha et al. (2000), $15=$ Boesgaard \& Friel (1990), 16= Balachandran (1990), 17 = Favata et al. (1997), 18 = Zboril \& Byrne (1998), $19=$ Cayrel de Strobel et al. (1989), $20=$ Fuhrmann (1998), $21=$ Balona et al. (1994), 22 = Malagnini \& Morossi (1990), 23 = Lebre et al. (1999), $24=$ Sokolov (1995), $25=$ Buscombe \& Morris (1960), $26=$ Söderhjelm (1999).

b $\mathrm{M}$ indicates the star is known to have multiple nearby companions which may be physically associated, B indicates the star is known to have one (possibly physical) companion, otherwise there is no entry. 
Table 2. Further properties of MONS short-listed stars. Column 1: Hipparcos No.; Cols. 2, 3, 4: $v \sin i, 1 \sigma$ error, source; 5, 6: Johnson $V$ magnitude, $1 \sigma$ error (Mermilliod et al. 1997); 7, 8: Johnson $B-V$ colour, $1 \sigma$ error (Mermilliod et al. 1997); 9, 10: visual extinction and uncertainty; Cols. 11, 12: luminosity and uncertainty; Cols. 13, 14: luminosity and uncertainty when setting $A_{V}=\sigma_{A}=0$.

\begin{tabular}{|c|c|c|c|c|c|c|c|c|c|c|c|c|c|}
\hline HIP & $\begin{array}{l}v \sin i \\
\quad[\mathrm{~km} s\end{array}$ & $\begin{array}{l}\sigma_{\mathrm{v}} \\
-1]\end{array}$ & ref. $^{a}$ & \multicolumn{2}{|c|}{$[\mathrm{mag}]$} & \multicolumn{2}{|c|}{ [mag] } & \multicolumn{2}{|c|}{ [mag] } & \multicolumn{2}{|c|}{$\left[L_{\odot}\right]$} & \multicolumn{2}{|c|}{$\begin{array}{c}L_{m} \quad \sigma_{L} \\
{\left[L_{\odot}\right]}\end{array}$} \\
\hline 2021 & 6.0 & 1.0 & 29 & 2.797 & 0.006 & 0.618 & 0.005 & 0.08 & 0.07 & 4.0 & 0.3 & 3.74 & 0.05 \\
\hline 3821 & 1.5 & 0.8 & 30 & 3.444 & 0.009 & 0.572 & 0.007 & 0.00 & 0.04 & 1.32 & 0.05 & 1.323 & 0.019 \\
\hline 7513 & 9.2 & 0.7 & 30 & 4.086 & 0.013 & 0.537 & 0.008 & 0.00 & 0.03 & 3.53 & 0.13 & 3.53 & 0.09 \\
\hline 8102 & 0.4 & 0.4 & 28 & 3.496 & 0.011 & 0.727 & 0.007 & 0.00 & 0.06 & 0.52 & 0.03 & 0.523 & 0.011 \\
\hline 8796 & 90. & 1.0 & 29 & 3.414 & 0.013 & 0.489 & 0.010 & 0.00 & 0.04 & 13.8 & 0.7 & 13.7 & 0.5 \\
\hline 12777 & 8.8 & 0.6 & 30 & 4.107 & 0.017 & 0.486 & 0.007 & 0.00 & 0.03 & 2.39 & 0.10 & 2.39 & 0.06 \\
\hline 14632 & 3.5 & 0.7 & 30 & 4.046 & 0.008 & 0.593 & 0.008 & 0.01 & 0.04 & 2.30 & 0.09 & 2.28 & 0.04 \\
\hline 14879 & 5.2 & 0.5 & 35 & 3.855 & 0.018 & 0.522 & 0.011 & 0.00 & 0.04 & 5.0 & 0.2 & 5.04 & 0.15 \\
\hline 15457 & 4.5 & 0.3 & 28 & 4.836 & 0.010 & 0.679 & 0.007 & 0.00 & 0.04 & 0.89 & 0.04 & 0.892 & 0.018 \\
\hline 16537 & 1.7 & 0.3 & 28 & 3.726 & 0.010 & 0.882 & 0.007 & 0.02 & 0.07 & 0.35 & 0.03 & 0.342 & 0.008 \\
\hline 17378 & 1.0 & 1.0 & 29 & 3.527 & 0.012 & 0.922 & 0.007 & 0.00 & 0.05 & 3.39 & 0.19 & 3.39 & 0.11 \\
\hline 19893 & 50. & & 36 & 4.242 & 0.004 & 0.309 & 0.005 & 0.01 & 0.03 & 6.4 & 0.2 & 6.39 & 0.15 \\
\hline 22449 & 18. & 1.5 & 30 & 3.188 & 0.006 & 0.450 & 0.008 & 0.00 & 0.03 & 2.77 & 0.09 & 2.77 & 0.05 \\
\hline 27072 & 8.7 & 0.8 & 32 & 3.591 & 0.013 & 0.480 & 0.012 & 0.00 & 0.03 & 2.45 & 0.08 & 2.45 & 0.05 \\
\hline 27913 & 9.4 & 0.4 & 30 & 4.401 & 0.010 & 0.591 & 0.010 & 0.00 & 0.03 & 1.13 & 0.04 & 1.13 & 0.02 \\
\hline 32362 & 70. & 7.0 & 23 & 3.350 & 0.014 & 0.437 & 0.006 & 0.00 & 0.04 & 11.4 & 0.6 & 11.4 & 0.4 \\
\hline 37279 & 6.1 & 1.0 & 29 & 0.367 & 0.010 & 0.421 & 0.008 & 0.00 & 0.03 & 7.0 & 0.2 & 7.04 & 0.10 \\
\hline 50954 & 50. & & 38 & 3.987 & 0.010 & 0.360 & 0.006 & 0.18 & 0.13 & 6.1 & 0.8 & 5.12 & 0.11 \\
\hline 55642 & 16. & 1.0 & 29 & 3.939 & 0.009 & 0.409 & 0.006 & 0.02 & 0.05 & 11.7 & 0.9 & 11.5 & 0.7 \\
\hline 57757 & 4.0 & 0.8 & 30 & 3.608 & 0.010 & 0.551 & 0.008 & 0.00 & 0.04 & 3.59 & 0.16 & 3.59 & 0.09 \\
\hline 67927 & 13. & 1.0 & 29 & 2.680 & 0.009 & 0.580 & 0.009 & 0.00 & 0.03 & 9.2 & 0.4 & 9.2 & 0.2 \\
\hline 70497 & 29. & 1.0 & 32 & 4.049 & 0.012 & 0.496 & 0.006 & 0.04 & 0.05 & 4.5 & 0.2 & 4.32 & 0.10 \\
\hline 71681 & 1.1 & 0.8 & 28 & 1.352 & 0.010 & 0.866 & 0.026 & 0.08 & 0.09 & 0.54 & 0.05 & 0.504 & 0.008 \\
\hline 71683 & 2.7 & 0.7 & 28 & 0.000 & 0.005 & 0.651 & 0.027 & 0.01 & 0.05 & 1.58 & 0.07 & 1.556 & 0.011 \\
\hline 71957 & 46. & & 34 & 3.876 & 0.012 & 0.382 & 0.005 & 0.19 & 0.09 & 9.0 & 0.9 & 7.5 & 0.3 \\
\hline 76976 & & & & 7.211 & 0.013 & 0.490 & 0.010 & 0.00 & 0.04 & 4.3 & 0.5 & 4.3 & 0.5 \\
\hline 77257 & 2.4 & 0.8 & 30 & 4.426 & 0.009 & 0.602 & 0.007 & 0.04 & 0.06 & 2.11 & 0.13 & 2.04 & 0.05 \\
\hline 78072 & 9.3 & 0.7 & 32 & 3.842 & 0.018 & 0.477 & 0.007 & 0.00 & 0.03 & 3.02 & 0.11 & 3.02 & 0.08 \\
\hline 81693 & 4.8 & 1.0 & 29 & 2.807 & 0.010 & 0.644 & 0.009 & 0.09 & 0.09 & 8.2 & 0.7 & 7.52 & 0.15 \\
\hline 84405 & 0.8 & 0.9 & 28 & 5.05 & 0.010 & 0.960 & 0.010 & 0.45 & 0.14 & 0.52 & 0.07 & 0.344 & 0.009 \\
\hline 86974 & 1.7 & 1.0 & 29 & 3.417 & 0.014 & 0.752 & 0.009 & 0.00 & 0.06 & 2.85 & 0.18 & 2.85 & 0.08 \\
\hline 88601 & 1.6 & 0.4 & 37 & 4.023 & 0.013 & 0.864 & 0.011 & 0.26 & 0.12 & 0.80 & 0.09 & 0.631 & 0.016 \\
\hline 89937 & 2.5 & 0.4 & 37 & 3.571 & 0.009 & 0.489 & 0.006 & 0.00 & 0.03 & 2.12 & 0.06 & 2.12 & 0.03 \\
\hline 99240 & & & & 3.556 & 0.011 & 0.760 & 0.002 & 0.04 & 0.08 & 1.33 & 0.10 & 1.29 & 0.03 \\
\hline 102485 & 41. & & 34 & 4.137 & 0.004 & 0.426 & 0.003 & 0.00 & 0.03 & 3.88 & 0.17 & 3.87 & 0.11 \\
\hline 104043 & 85. & & 38 & 5.140 & 0.008 & 0.488 & 0.007 & 0.03 & 0.10 & 15.1 & 1.7 & 14.8 & 0.9 \\
\hline 105858 & 8. & & 33 & 4.213 & 0.009 & 0.484 & 0.006 & 0.01 & 0.03 & 1.56 & 0.05 & 1.55 & 0.03 \\
\hline 109176 & 6.5 & 0.8 & 30 & 3.768 & 0.006 & 0.433 & 0.008 & 0.00 & 0.03 & 3.51 & 0.12 & 3.51 & 0.07 \\
\hline 110618 & 0.0 & & 31 & 5.279 & 0.007 & 0.657 & 0.010 & 0.05 & 0.09 & 6.7 & 0.6 & 6.4 & 0.2 \\
\hline 114996 & 80. & 5.0 & 16 & 3.985 & 0.005 & 0.400 & 0.006 & 0.00 & 0.03 & 10.1 & 0.4 & 10.1 & 0.3 \\
\hline
\end{tabular}

a Refs. (in addition to Table 1): 28 = Saar \& Osten (1997), $29=$ de Medeiros et al. (1997), $30=$ Soderblom (1982), $31=$ Andersen et al. (1984), $32=$ Soderblom et al. (1989), $33=$ Schrijver (1993), $34=$ Simon \& Drake (1989), $35=$ Hale (1994), $36=$ Soderblom (1983), $37=$ Gray (1984), 38 = Uesugi \& Fukuda (1992).

In such cases the $A_{V}$ is set to 0 in Table 2 . In all but a few of the cases where the $A_{V}$ is set to 0 , the value is between 0 and $-1.5 \sigma$. For the exceptions the absolute value of the extinction $\left|A_{V}\right|<3 \sigma$. These exceptions are:

- HIP 37279 ( $\alpha C M i A)$ : it is possible that the measured ( $B$ $V)$ is affected by the presence of the white dwarf companion (Procyon B);

- HIP 17378 ( $\delta$ Eri): this star is classified as an RS CVn type variable (a detached active binary) which could be the cause for anomalous colours;
- HIP 89937 ( $\chi$ Dra): An astrometric, interferometric, and spectroscopic binary. If the secondary is also a main sequence star it should not have a higher $T_{\text {eff }}$ in which case it would not be likely to cause an anomalously blue colour of the combined binary;

- HIP 109176 ( ( Peg A): A spectroscopic binary with a period of $\sim 10 \mathrm{~d}$. Possibly the companion is sufficiently near in mass to affect the colour.

As expected for nearby stars, for all stars in Table 2 but one the value for $\left|A_{V}\right|<3 \sigma\left(A_{V}\right)$. The one exception is 36 Oph A 


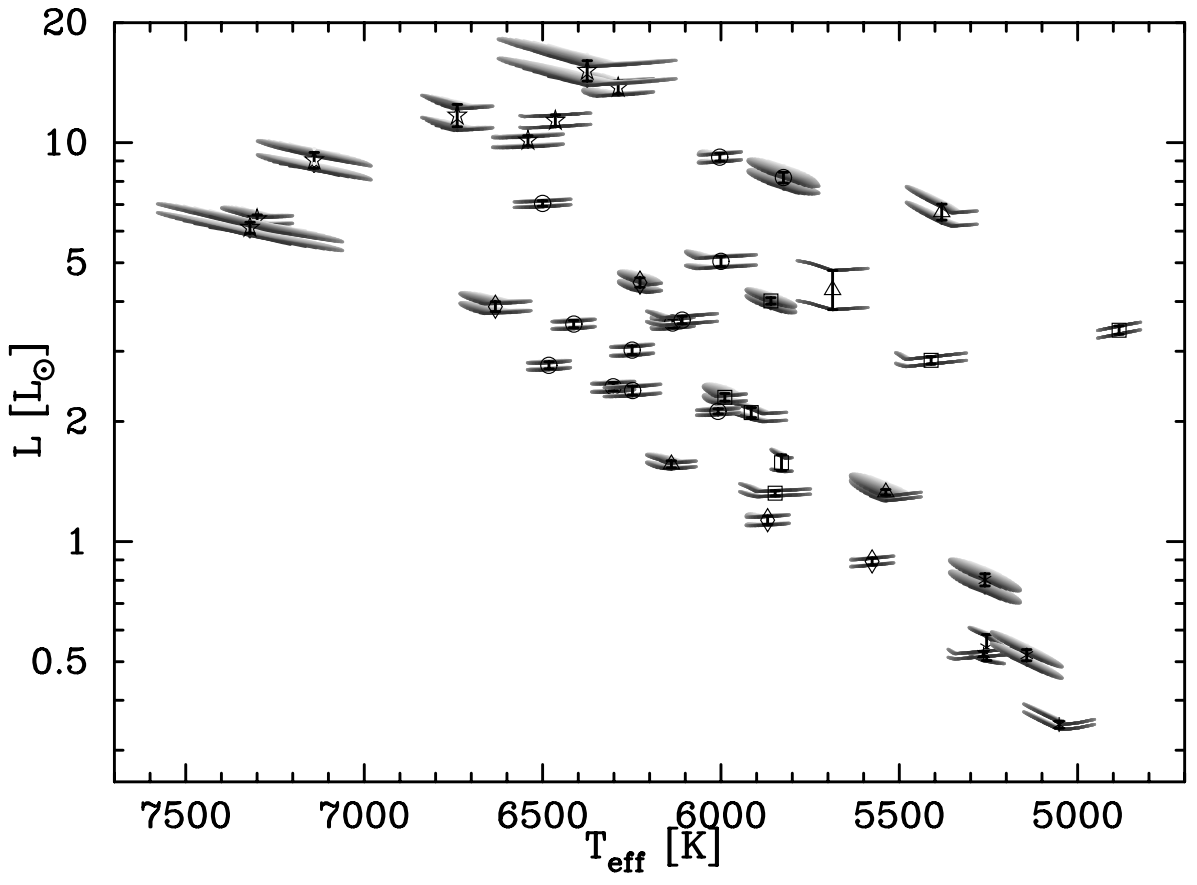

Fig. 1. The theoretical HR diagram of the MONS short-listed stars. Symbols are: squares: solar mass, asterisks: lower mass, circles: higher mass, triangles: lower and higher metallicity, diamonds: rapid rotator, stars: hotter. For each star is shown the upper and lower $1 \sigma$ error bound around the luminosity for the appropriate $T_{\text {eff }}$ within its $1 \sigma$ error. The shading from light to dark of each elliptical surface corresponds to $[\mathrm{Fe} / \mathrm{H}]$ running from the lowest to the highest value within its $1 \sigma$ error bound.
(HIP 84405) which has a nearly equal mass companion so that for this star the determination of $A_{V}$ is probably an overestimate. It is important for high precision photometry to know whether or not a target star has one or more close companions, for instance because it is member of a (close) binary or multiple system, which is why this is indicated in Table 2. One star for which binarity is indicated is $v$ Ind (HIP 110618). However there is some doubt as to the binarity of this star (cf. Lambert \& McWilliam 1986) and evidently the spectral type is not consistent with the spectroscopic effective temperature.

The uncertainty in the luminosity in Table 2 is obtained by treating each of the terms in Eq. (2) as a statistically independent source of errors and combining the variances appropriately. Although the number obtained in this way does provide a measure of the range of luminosities consistent with the data, it is misleading in that the error distribution is not Gaussian since there is a correlation between e.g. the effective temperature and the bolometric correction on $V$. In the theoretical HR diagram Fig. 1 account is taken of this by showing for each star the $1 \sigma$ lower and upper bound for the luminosity obtained for $T_{\text {eff }}$ and $[\mathrm{Fe} / \mathrm{H}]$ within their combined $1 \sigma$ error bounds. In practice in all cases the largest remaining source of error is due to the uncertainty in $A_{V}$ which arises in most cases primarily from the $1 \sigma$ error on the observed $B-V$ colour. In Table 2 the minimum luminosity $L_{\mathrm{m}}$ and its uncertainty for each star are also given, calculated as above but setting both $A_{V}=0$ and $\sigma_{A}=0$. The uncertainty $\sigma_{L}$ obtained in this way has roughly equal contributions from the errors in the $V$ magnitude, the parallax, and the bolometric correction.

From Fig. 1 it is seen that the sample covers a range in masses and ages which is essential for the purposes of testing stellar evolution theory. In Fig. 1 each star is represented by two elliptical surfaces, in some cases with a "kink", connected by a vertical bar at the $T_{\text {eff }}$ listed in Table 1 . The bolometric correction depends on $T_{\text {eff }}$, and $[\mathrm{Fe} / \mathrm{H}]$ and to a lesser extent on $\log g$, which means that the $1 \sigma$ range for the luminosity is not identical for every combination of $T_{\text {eff }}$ and $[\mathrm{Fe} / \mathrm{H}]$ within their combined $1 \sigma$ error ellipse. For each star the upper ellipse represents the $1 \sigma$ upper limit for the luminosity and the lower ellipse represents the $1 \sigma$ lower limit. The shading of each ellipse corresponds to the $[\mathrm{Fe} / \mathrm{H}]$ running from the central $-1 \sigma$ to $+1 \sigma$ value. For some stars the latter source of uncertainty is so small that the ellipses reduce to lines. The "kinks" can occur because the intrinsic colour of the star becomes bluer, $(B-V)_{0}$ smaller, as the effective temperature is higher. From Eq. (2) it can be seen that for $T_{\text {eff }}$ higher than a certain value $A_{V}$ becomes positive and therefore the inferred luminosity after correcting for this extinction increases towards higher $T_{\text {eff }}$, whereas for lower $T_{\text {eff }} A_{V}$ remains fixed at 0 since a negative extinction is unphysical.

As is discussed in Sect. 1, the aims of asteroseismic campaigns include obtaining masses and rotation rates of stars with higher precision than currently available. It is therefore of interest to show diagrams for the mass and the angular momentum of these stars which can be determined directly, even if only with low precision and up to a factor $\sin i$ in the unknown inclination angle, from the data listed in Tables 1 and 2. In Fig. 2 the angular momentum is shown versus the stellar mass. The (spectroscopic) mass of the stars can be estimated using:

$\log \frac{M}{M_{\odot}}=\log \frac{g}{g_{\odot}}+\log \frac{L}{L_{\odot}}-4 \log \frac{T_{\text {eff }}}{T_{\text {eff } \odot}}$.

Similarly the (spectroscopic) angular momentum $H$ can be estimated as follows:

$$
\begin{aligned}
\log \frac{H \sin i}{H_{\odot}}= & \log \frac{g}{g_{\odot}}+\frac{3}{2} \log \frac{L}{L_{\odot}}-6 \log \frac{T_{\text {eff }}}{T_{\text {eff } \odot}} \\
& +\log I_{M}+\log \frac{v \sin i}{v_{\odot}} .
\end{aligned}
$$

However, for a few stars a dynamic mass $M_{\text {dyn }}$ is available which is preferable to use since it is always more accurate. 
Table 3. Dynamic masses of some of the MONS short-listed stars.

\begin{tabular}{|c|c|c|c|}
\hline HIP No. & $\bar{M}$ & $\begin{array}{l}\sigma_{M} \\
\odot]\end{array}$ & ref. $^{a}$ \\
\hline 3821 & 0.91 & 0.05 & 1 \\
\hline 27913 & 1.02 & 0.08 & 7 \\
\hline 37279 & 1.497 & 0.037 & 2 \\
\hline 55642 & 1.7 & 0.2 & 5 \\
\hline 71681 & 0.934 & 0.0061 & 3 \\
\hline 71683 & 1.105 & 0.0070 & 3 \\
\hline 81693 & 1.25 & 0.05 & 1 \\
\hline 88601 & 0.90 & 0.074 & 4 \\
\hline 89937 & 0.98 & 0.03 & 5 \\
\hline 109176 & 1.31 & 0.02 & 6 \\
\hline
\end{tabular}

a Refs.: 1 = Harmanec (1988), 2 = Girard et al. (2000), 3 = Pourbaix et al. (2002), 4= Pourbaix (2000), 5= Söderhjelm (1999), $6=$ Fekel \& Tomkin (1983), 7 = König et al. (2002).

In this case the appropriate equation for $H$ is:

$$
\begin{aligned}
\log \frac{H \sin i}{H_{\odot}}= & \log \frac{M_{\mathrm{dyn}}}{M_{\odot}}+\frac{1}{2} \log \frac{L}{L_{\odot}}-2 \log \frac{T_{\mathrm{eff}}}{T_{\mathrm{eff}, \odot}} \\
& +\log I_{M}+\log \frac{v \sin i}{v_{\odot}}
\end{aligned}
$$

Those stars in the MONS short-list for which dynamic masses are available are summarized in Table 3.

In Eqs. (4) and (5) the term $I_{M}$ is the scaled moment of inertia of the star:

$I_{M}=\frac{8 \pi}{3 M R^{2}} \int_{0}^{R} \rho r^{4} \mathrm{~d} r$.

The total angular momentum of a star is an integral over the stellar interior of the angular momentum per unit mass, i.e. a density weighted average of the rotation rate $\Omega(r, \theta)$. If the stars were to rotate uniformly the above equations would hold exactly. For the Sun the true angular momentum is known to be $H_{\odot}=190 \pm 1.5 \times 10^{39} \mathrm{~kg} \mathrm{~m}^{2} \mathrm{~s}^{-1}$ (Pijpers 1998) which is determined helioseismically taking into account the full radial and latitudinal dependence of the rotation rate $\Omega_{\odot}(r, \theta)$. Using this value in Eq. (5), a value for $I_{M}$ calculated from a standard solar model (cf. Christensen-Dalsgaard et al. 1996), and using the observed equatorial surface rotation velocity $v_{\odot}=1.93 \mathrm{~km} \mathrm{~s}^{-1}$ the deviation from a value of 1 is less than $2 \%$, because the deviations from uniform rotation in the Sun are small. However, even if the interior density of a star can be calculated from models, the interior rotation rate of stars other than the Sun is still unknown. Therefore in producing Fig. 2, using Eqs. (4) or (5), the value of $I_{M}$ is kept fixed at $I_{M_{\odot}}$. For stellar models the value of $I_{M} / I_{M_{\odot}}$ decreases with increasing mass and with increasing age on the main sequence. In the mass range of 0.85 to 4.0 solar masses, and with ages spanning the main sequence lifetime, this ratio lies between 0.5 and 2.0. Including this in Fig. 2 would have the effect of making the trend slightly less steep. In order to be able to show error bars on the angular momentum estimated using Eqs. (4) or (5) for those stars in Table 2 for which there is no error estimate for $v \sin i, \sigma_{v}$ is set

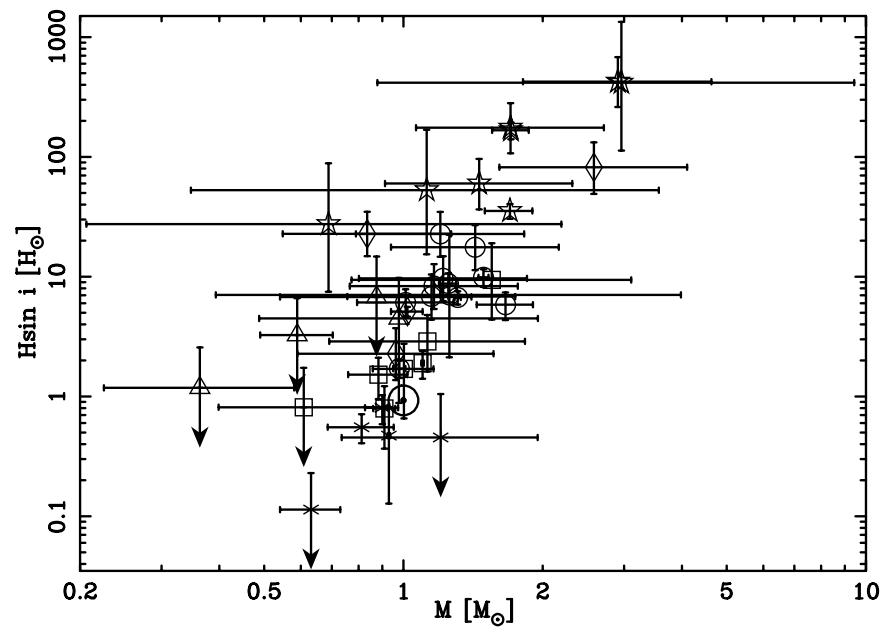

Fig. 2. Angular momentum versus mass for the MONS short-listed stars. Symbols as in Fig. 1. The symbol $\odot$ is for the Sun, located away from unity due to differential rotation as explained in the text.

to $5.0 \mathrm{~km} \mathrm{~s}^{-1}$. For the stars for which no determination of $v \sin i$ is available $v \sin i$ and $\sigma_{v}$ are both set to $5.0 \mathrm{~km} \mathrm{~s}^{-1}$ as well. In the latter case the value is an upper limit, which is indicated by downward arrows in Fig. 2. The "rapid rotators" do not appear to stand out significantly from the general trend in Fig. 2. Clearly for any significant determination of a dependence of angular momentum on mass or age, a much higher precision for both mass and angular momentum is required. A precision similar to that of the dynamical masses in Table 3 is realistic to expect from asteroseismic data to be collected by the various satellite missions.

\section{The selection criteria}

The focus of the MONS asteroseismic mission is on solar-like stars for two reasons: firstly it is important for the purposes of mode identification as well as for the quality of the asteroseismic inferences to be made that many modes of pulsation be excited simultaneously. The highest probability for this to occur is in stars where the oscillations are excited by convection such as the Sun, because in principle this can excite all possible modes contrary to other excitation mechanisms. The second reason for choosing stars not too dissimilar from the Sun is that asteroseismic inference is still in its infancy. The likelihood of success in interpreting asteroseismic data is higher for stars that are in most respects close analogues of the Sun. The MONS short-listed stars span the range of $T_{\text {eff }}$ for which this can be said to be the case. Spectroscopically obtained values (and their uncertainties!) for $\left(T_{\mathrm{eff}}, \log g,[\mathrm{Fe} / \mathrm{H}]\right)$ are considered to be essential in the determination of high quality stellar parameters, rather than relying on colour-calibrations. One of the criteria to be used in forming a sample of stars is that such spectroscopy has been done. Unfortunately with currently available data this leads to a biased sample of stars. It would be desirable to be able to have spectroscopy for a volume-limited sample of stars but this requires a considerable investment both observationally and in terms of data analysis and interpretation. 
The driving factor for the selection criteria is to obtain useful estimates of the bolometric luminosity from the observed data, since it is this parameter together with the effective temperature which are currently used as constraints for stellar evolution models. In order to be able to do photometry at the ppm level with a spaceborn telescope with an collecting area of $\sim 600 \mathrm{~cm}^{2}$ (i.e. MONS), the stars must be bright. However, the space density of metal poor stars is quite low which means that any sample which does not differentiate in metallicity will contain very few metal-poor stars. In order to obtain a reasonably large sample of Pop. I and II stars, a separate magnitude limit for each group appears desirable. At the same luminosity a fainter magnitude limit corresponds to larger distances. Two different criteria for the parallax are therefore proposed as well. In the MONS short-list of potential targets, the limiting factor on the precision of the luminosity is the uncertainty in $A_{V}$ which is typically around 0.06 . Setting the estimated error due to the error in the parallax to be maximally the same value implies that $\pi / \sigma(\pi)>36.2$. The best parallax determinations from the Hipparcos catalogue have $\sigma(\pi)=0.45$ mas so that no stars with a parallax $\pi<16.2$ mas would qualify.

The quantitative selection criteria for suitable targets for the MONS mission can now be summarized as follows:

- Select stars for which spectroscopic determinations of $\left(T_{\mathrm{eff}}, \log g,[\mathrm{Fe} / \mathrm{H}]\right)$ are available (e.g. from Cayrel de Strobel et al. 2001) and for which $4850 \mathrm{~K}<$ $T_{\text {eff }}<7350 \mathrm{~K}$;

- Two separate brightness criteria are proposed: Group (a) stars, for which $[\mathrm{Fe} / \mathrm{H}]<-0.6$, pass if their $V$ magnitude satisfies $V<7.3$. Group (b) stars pass if their $V$ magnitude satisfies $V<5.3$. The corresponding noise level ( $4 \sigma$ detection limit in amplitude after 30 days observing with a duty cycle of $85 \%$ for the faintest stars in the group) of the photometry for the MONS mission is $\sim 13 \mathrm{ppm}$ for group (a), and $\sim 4.5$ ppm for group (b) (Kjeldsen \& Bedding 2001);

- The two separate distance criteria for group (a) and (b), corresponding in space volume to the two separate limiting magnitudes, are: the group (a) of stars for which $[\mathrm{Fe} / \mathrm{H}]<-0.6$ pass if their parallax satisfies $\pi / \sigma(\pi)>14$ (which implies $\pi>6.4$ mas), and the group (b) of stars pass if their parallax satisfies $\pi / \sigma(\pi)>36$ (which implies $\pi>16.2$ mas).

By selection from the Hipparcos catalogue (ESA 1997) there are 407 stars which pass the criteria $V<5.3$ and $\pi / \sigma(\pi)>36$ (group (b)), but there is good spectroscopy currently available in the literature for only 196 of them. For the selection of stars for group (a) $(V<7.3$ and $\pi / \sigma(\pi)>14)$ there are 2428 additional candidates from the Hipparcos catalogue but in order to apply the metallicity criterion good spectroscopy must be available, so it is unclear how many of these stars would in fact qualify. The number of stars for which good spectroscopy is available which pass all the criteria for group (a), including the metallicity criterion, currently stands at 36 .

It should be noted that still not all stars selected in this way are suitable for performing asteroseismology. The satellite missions are dependent on doing high precision time-series photometry, and the presence of possibly variable background stars within arcmin of the target can have serious detrimental effects on the signal-to-noise ratio. The stellar census from surveys is usually incomplete very near to bright stars because of saturation effects. Therefore dedicated imaging of the near field of potential targets has been or is being undertaken for the COROT and MONS missions. Furthermore, for a mission such as COROT which will select a very small set of fields in order to obtain very long time-series, the selected fields should preferably have within them a number of unsaturated, bright stars representing as wide a range of $\left(T_{\text {eff }}, \log g,[\mathrm{Fe} / \mathrm{H}]\right)$ as possible, which is a more complex optimization problem.

\section{Conclusions}

For those stars that are the main targets for the MONS experiment on board the planned Danish small satellite Rømer, the luminosity is rederived using the BaSeL library of model atmospheres (Lejeune et al. 1998). It is shown that even for these stars, the brightest in their respective classes, the uncertainty in their fundamental parameters is still substantial in some cases and it is worthwhile obtaining, or re-analysing, high quality spectra. It is also clear that for single stars the direct constraint on stellar masses from spectroscopy is weak. The constraints on angular momenta of stars from spectroscopy are weak as well since there is little knowledge of the internal rotation of stars and there is always the $\sin i$ ambiguity. For binaries one might assume that the rotation axes are perpendicular to the orbital plane, but this implies a certain exchange of angular momentum between the star and the orbit and therefore a bias is introduced. This implies that the angular momentum distribution and evolution of field stars in the galaxy is poorly constrained. It is here that asteroseismology can provide considerable improvement through determination of stellar masses (cf. Christensen-Dalsgaard 1993) and also stellar angular momenta (Pijpers 2003).

Using the present set as a template, a set of objective sample selection criteria for stars of spectral type F, G, and K can be defined. It is clear that there are several hundred stars for which asteroseismology from space could potentially provide strong constraints on stellar structure and evolution modelling. However, with currently available spectroscopic data the full potential can be exploited for less than half of those stars. In preparation for the ESA space mission Eddington it is desirable to carry out extensive ground-based programs of highresolution high S/N spectroscopy for these stars, and to do detailed stellar atmosphere modelling in order to obtain precise fundamental parameters.

Acknowledgements. The author thanks Jørgen ChristensenDalsgaard, Hans Bruntt, Hans Kjeldsen, and Teresa Teixeira for many useful comments and discussions, and the referee R. Garrido for suggestions improving the presentation of the paper. The author also thanks the Theoretical Astrophysics Centre, a collaborative centre between Copenhagen University and Aarhus University funded by the Danish Research Foundation for support of this work. This research has made use of the VizieR catalogue access tool, CDS, Strasbourg, France. 


\section{References}

Abia, C., Rebolo, R., Beckman, J. E., \& Crivellari, L. 1988, A\&A, 206, 100

Abt, H. A., \& Levy, S. G. 1976, ApJS, 30, 273

Andersen, J., Gustafsson, B., \& Lambert, D. L. 1984, A\&A, 136, 65

Asplund, M., Nordlund, A., Trampedach, R., \& Stein, R. F. 1999 A\&A, 346, L17

Balachandran, S. 1990, ApJ, 354, 310

Balona, L. A., Hearnshaw, J. B., Koen, C., et al. 1994, MNRAS, 267, 103

Bessel, M. S., Castelli, F., \& Plez, B. w. 1998, A\&A, 333, 231

Boesgaard, A. M., \& Friel, E. D. 1990, ApJ, 351, 467

Brown, T. M., Christensen-Dalsgaard, J., Weibel-Mihalas, B., \& Gilliland, R. L. 1994, ApJ, 427, 1013

Bruntt, H., in preparation

Buscombe, W., \& Morris, P. M. 1960, Observatory, 80, 28

Canuto, V. M., \& Dubovikov, M. 1998, ApJ, 493, 834

Castro, S., Porto de Mello, G. F., \& da Silva, L. 1999, MNRAS, 305, 693

Cayrel de Strobel, G., Lebreton, Y., Perrin, M.-N., \& Cayrel, R. 1989, A\&A, 225, 369

Cayrel de Strobel, G., Soubiran, C., \& Ralite, N. 2001, A\&A, 373, 159

Cenarro, A. J., Cardiel, N., Pedraz, S., Peletier, R. F., \& Vazdekis, A. 2001, MNRAS, 326, 981

Christensen-Dalsgaard, J. 1993, Proc. GONG 1992, seismic investigation of the Sun and stars, ed. T. M. Brown, PASP Conf. Ser., 42, 347

Christensen-Dalsgaard, J., Däppen, W., Ajukov, S. V., et al. 1996, Science, 272, 1286

Christensen-Dalsgaard, J. 2001, Rømer science mission specification, 52

Christensen-Dalsgaard, J. 2002, in Proc. 1st Eddington workshop, Stellar structure and habitable planet finding, ed. F. Favata, I. W. Roxburgh, \& D. Galadi, ESA, 25

Cunha, K., Smith, V. V., Boesgaard, A. M., \& Lambert, D. L. 2000, ApJ, 530, 939

ESA 1997, The Hipparcos and Tycho Catalogues

Favata, F., Micela, G., \& Sciortino, S. 1997, A\&A, 323, 809

Fekel, F. C., \& Tomkin, J. 1983, PASP, 95, 1000

Fuhrmann, K. 1998, A\&A, 338, 161

Girard, T. M., Wu, H., Lee, J. T., et al. 2000, AJ, 119, 2428

Gratton, R. G., Sneden, C., Carretta, E., \& Bragaglia, A. 2000, A\&A, 354,169
Gray, D. F. 1984, ApJ, 281, 719

Halbwachs, J. L. 1981, A\&AS, 44, 47

Hale, A. 1994, AJ, 107, 306

Harmanec, P. 1988, Bull. Astron. Inst. Czech., 39, 329

Hoffleit, D., \& Warren, Jr. W. H. 1991, The Bright Star Catalogue, 5th revised edn.

Kjeldsen, H., \& Bedding, T. 2001, The MONS payload requirements specification, 19

König, B., Fuhrmann, K., Neuhäuser, Charbonneau, D., \& Jayawardhana, R. 2002, A\&A, 394, L43

Lambert, D. L., \& McWilliam, A. 1986, ApJ, 304, 436

Lebre, A., de Laverny, P., de Medeiros, J. R., Charbonnel, C., \& da Silva, L. 1999, A\&A, 345, 936

Lejeune, T., Cuisinier, F., \& Buser, R. 1998, A\&AS, 130, 65

Malagnini, M. L., \& Morossi, C. 1990, A\&AS, 85, 1015

Mashonkina, L., \& Gehren, T. 2000, A\&A, 364, 249

de Medeiros, J. R., Do Nascimento, Jr. J. D., \& Mayor, M. 1997, A\&A, 317,701

Mermilliod, J.-C., Mermilliod, M., \& Hauck, B. 1997, A\&AS, 124, 349

Neckel, Th., Klare, G., \& Sarcander, M. 1980, A\&AS, 42, 251

Neuforge-Verheecke, C., \& Magain, P. 1997, A\&A, 328, 261

Pijpers, F. P. 1998, MNRAS, 297, L76

Pijpers, F. P. 2003, Proc. Asteroseismology Across the HR Diagram, ed. M. J. Thompson, M. S.Cunha, \& M. J. P. F. G. Monteiro (Kluwer)

Pourbaix, D. 2000, A\&AS, 145, 215

Pourbaix, D., Nidever, D., McCarthy, C., et al. 2002, A\&A, 386, 280

Saar, S. H., \& Osten, R. H. 1997, MNRAS, 284, 803

Schrijver, C. J. 1993, A\&A, 269, 446

Simon, T., \& Drake, S. A. 1989, ApJ, 346, 303

Soderblom, D. R. 1982, ApJ, 263, 239

Soderblom, D. R. 1983, ApJS, 53, 1

Soderblom, D. R., Pendleton, J., \& Pallavicini, R. 1989, AJ, 97, 539

Sokolov, N. A. 1995, A\&AS, 110, 553

Soubiran, C., Katz, D., \& Cayrel, R. 1998, A\&AS, 133, 221

Söderhjelm, S. 1999, 341, 121

Uesugi, A., \& Fukuda, I. 1992, Revised Catalogue of Stellar Rotational Velocities

Worley, C. E., \& Douglass, G. G. 1996, The Washington Visual Double Star Catalog, A\&AS, 125, 523

Zboril, M., \& Byrne, P. B. 1998, MNRAS, 299, 753 